\title{
ANALYTIC ULTRADISTRIBUTIONS
}

\author{
B. STANKOVIĆ
}

(Communicated by Palle E. T. Jorgensen)

\begin{abstract}
A necessary and sufficient condition that an ultradistribution, of Beurling or Roumieu type, which is defined on an open set $\Omega \subset \mathscr{R}^{n}$ is a real analytic function is given. This result is applied to different problems.
\end{abstract}

\section{INTRODUCTION}

It is of interest to know whether a generalized function $T$ or its restriction to an open set is defined by a real analytic function, especially if this generalized function is a solution of an equation. One can find answers for different classes of generalized functions in [2], [3] and [5]. An answer on this question for distributions was made by L. Schwartz (see [8, Chapter VI, Theorem XXIV]). Our aim is to extend this result to ultradistributions. The key of the proof of the mentioned theorem in [8] was in the parametrix. Therefore we cannot proceed analogously to answer this question for ultradistributions. In our proof we use an assertion which can be found in Komatsu [4] and applied also by Pilipović [7]. We cite this assertion as Theorem A.

As an illustration of applications of Theorem 1 we give two direct consequences and two theorems concerning convolution equations and Painlevé's theorem.

\section{NotATION}

We follow the notation of [6]. Let us repeat some.

By $M_{p}$ we denote a sequence of positive numbers satisfying some of the following conditions: $M_{0}=M_{1}=1$ and

(M.1) $M_{p}^{2} \leq M_{p-1} M_{p+1}, p \in \mathscr{N}$;

(M.2) $M_{p} /\left(M_{q} M_{p-q}\right) \leq A B^{p}, 0 \leq q \leq p, p, q \in \mathscr{N}$;

(M.3) $\sum_{q=p+1}^{\infty} M_{q-1} / M_{q} \leq A p M_{p} / M_{p+1}, p \in \mathscr{N}$,

where $A$ and $B$ are constants independent on $p$.

We use two classes of ultradifferentiable functions, the Beurling class and Reumieu class (in short, $\left(M_{p}\right)$ class and $\left\{M_{p}\right\}$ class).

Let $u$ be a positive number and $\left\{u_{p}\right\}$ be a sequence of positive numbers

Received by the editors April 13, 1994.

1991 Mathematics Subject Classification. Primary 46F05; Secondary 46F10. 
increasing to $\infty$. We denote

$$
H_{p}=\left\{\begin{array}{cc}
u^{p}, & \text { for the class }\left(M_{p}\right) \\
u_{1} \cdots u_{p}, & \text { for the class }\left\{M_{p}\right\}
\end{array}\right\} .
$$

Let $\Omega$ be an open set in $\mathscr{R}^{n}$.

By $\mathscr{D}_{K}^{H_{p} M_{p}}$ is denoted the Banach space of all $f \in \mathscr{C}^{\infty}\left(\mathscr{R}^{n}\right)$ with support in $K$ such that

$$
\sup _{x \in K}\left|f^{(p)}(x)\right| / H_{|p|} M_{|p|} \rightarrow 0 \quad \text { as } p \rightarrow \infty
$$

with the norm

$$
q_{H_{p} M_{p}}(f)=\sup _{x \in K, p \in \mathcal{N}_{0}^{n}}\left|f^{(p)}(x)\right| / H_{|p|} M_{|p|},
$$

where $K$ is a compact set in $\mathscr{R}^{n}$. Then the basic spaces are

$$
\mathscr{D}_{K}^{\left(M_{p}\right)}=\operatorname{proj} \lim _{u \rightarrow 0} \mathscr{D}_{K}^{u^{p} M_{p}}, \quad \mathscr{D}_{K}^{\left\{M_{p}\right\}}=\operatorname{proj} \lim _{H_{p}} \mathscr{D}_{K}^{H_{p} M_{p}}
$$

and

$$
\mathscr{D}^{*}(\Omega)=\text { ind } \lim _{K \in \Omega} \mathscr{D}_{K}^{*},
$$

where $*$ denotes either $\left(M_{p}\right)$ or $\left\{M_{p}\right\}$.

An operator of the form

$$
P(D)=\sum_{|i| \geq 0} a_{i} D^{i}, \quad a_{i} \in \mathscr{C},
$$

is called the ultradifferential operator of class $\left(M_{p}\right)$ (of class $\left\{M_{p}\right\}$ ) if there are constants $L$ and $C$ (for every $L>0$ there is a constant $C$ ) such that $\left|a_{i}\right| \leq C L^{|i|} / M_{|i|}, i \in \mathscr{N}_{0}^{n}$.

$A(\Omega)$ is the space of real analytic functions.

$B_{r} \equiv B(0, r)$ is the open ball with center at zero and with radius $r$. .

Theorem $A$ (see [4, Theorem 2.11]). Let the sequence $M_{p}$ satisfy conditions (M.1), (M.2) and (M.3). For a given $H_{p}$ from (1) and a compact neighbourhood $Q$ of zero in $\mathscr{R}^{n}$ there exist an ultradifferential operator $P(D)$ of class * and two functions $\varphi \in \mathscr{C}^{\infty}$ and $w \in D_{Q}^{*}$ such that

$$
P(D) \varphi=\delta+w, \quad \operatorname{supp} \varphi \subset Q
$$

and

$$
\sup _{x \in Q}\left|\varphi^{(i)}(x)\right| / H_{|i|} M_{|i|} \rightarrow 0, \quad|i| \rightarrow \infty .
$$

\section{MAIN RESULT}

Theorem 1. Suppose that $M_{p}$ satisfies (M.1), (M.2) and (M.3) and that $\Omega, \Omega_{1}$ are two open sets in $\mathscr{R}^{n}$ such that $\Omega=\Omega_{1}-B_{r}$ for some $r>0$. An ultradistribution $T \in \mathscr{D}^{\prime *}(\Omega)$ is defined by the real analytic function $f, f \in \mathscr{A}(\Omega)$, if and only if $T \star w \in \mathscr{A}\left(\Omega_{1}\right)$ for every $w \in \mathscr{D}_{K}^{*}$, where $K=\bar{B}_{r}$ and $\star$ is the sign of convolution.

Proof. If $T=f \in \mathscr{A}(\Omega)$, then it can be characterized as an infinitely differentiable function such that for every compact set $K^{\prime} \subset \Omega$ and $p \in \mathscr{N}_{0}^{n}$ there exist two constants $C_{K^{\prime}}$ and $C$ for which

$$
\sup _{x \in K^{\prime}}\left|f^{(p)}(x)\right| \leq C p ! C_{K^{\prime}}^{|p|}, \quad p \in \mathscr{N}_{0}^{n} .
$$


Take any compact set $K^{\prime \prime} \subset \Omega_{1}$. Then

$$
\sup _{x \in K^{\prime \prime}}\left|(f \star w)^{(p)}(x)\right| \leq C p ! C_{K^{\prime}}^{|p|} \int_{\mathscr{R}^{n}}|w(x)| d x, \quad p \in \mathscr{N}_{0}^{n},
$$

which proves that $f \star w \in \mathscr{A}\left(\Omega_{1}\right)$.

Suppose now that for $T \in \mathscr{D}^{\prime *}(\Omega)$ and for every $w \in \mathscr{D}_{K}^{*}, T \star w \in \mathscr{A}\left(\Omega_{1}\right)$ and $A$ is an open and relatively compact set, $\bar{A} \subset \Omega \subset \Omega_{1}$. In the first step of the proof we shall analyse the functional $R$,

$$
R:(w, q) \rightarrow \sup _{x \in \bar{A}}|q| \sqrt{\left|(f \star w)^{(q)}(x)\right| / q !}, \quad w \in \mathscr{D}_{K}^{*}, q \in \mathscr{N}^{n},
$$

which is related to the convergence radii of the power series

$$
\sum_{|q| \geq 0}(f \star w)^{(q)}(x)(x-y)^{q} / q !, \quad x \in A .
$$

For a fixed $q \in \mathscr{N}^{n}, R$ is continuous in $w \in \mathscr{D}_{K}^{*}$, and for a fixed $w \in \mathscr{D}_{K}^{*}$, $R$ is bounded in $q \in \mathscr{N}^{n}$. Since $\mathscr{D}_{K}^{*}$ is barreled space, by the Banach theorem it follows that the family $\left\{R(\cdot, q), q \in \mathscr{N}^{n}\right\}$ is equicontinuous. Then for a fixed $L>0$ there exist $\beta>0$ and $H_{p}$ such that $R(w, q)<L$ when $w \in V_{\beta}$ and $q \in \mathscr{N}^{n}$, where $V_{\beta}$ is the neighbourhood of zero in $\mathscr{D}_{K}^{*}$ of the form

$$
V_{\beta}=\left\{\phi \in \mathscr{D}_{K}^{*}: \sup _{x \in K, p \in \mathcal{N}_{0}^{n}}\left|\phi^{(p)}(x)\right| / H_{|p|} M_{|p|}<\beta\right\} .
$$

From the properties of the functional $R$ it follows that the family of functions

$$
F_{q}: w \rightarrow(T \star w)^{(q)} / q ! L^{|q|}=\left(D^{q} T \star w\right) / q ! L^{|q|}, \quad q \in \mathscr{N}_{0}^{n},
$$

is equicontinuous; $F_{q}$ maps $\mathscr{D}_{K}^{*}$ into $(\mathscr{C} \mathscr{B})_{A} .(\mathscr{C} \mathscr{B})_{A}$ is the space of continuous and bounded functions on $A$. Also, for every $w \in V_{\beta}$ and for every $q \in \mathscr{N}_{0}^{n},(T * w)^{(q)} / q ! L^{|q|}$ belongs to the ball $B(0, L) \subset(\mathscr{C} \mathscr{B})_{A}$. Namely, there exists $C>0$ such that

$$
\left|(T \star w)^{(q)}(x)\right| \leq C q ! L^{|q|}, \quad x \in \bar{A}, w \in V_{\beta} .
$$

Denote by $\widetilde{\mathscr{D}}_{K}^{H_{p} M_{p}}$ the completion of $\mathscr{D}_{K}^{*}$ under the norm $q_{H_{p} M_{p}}$ given by (2), where $H_{p}$ is fixed by $V_{\beta}$. In the second part of the proof we shall show that the family $\left\{F_{q}: q \in \mathcal{N}_{0}^{n}\right\}$ can be extended by $\mathscr{D}_{K}^{*}$ to $\widetilde{\mathscr{D}}_{K}^{H_{p} M_{p}}$, keeping uniform continuity; let us denote this extension by $\left\{\bar{F}_{q}: q \in \mathscr{N}_{0}^{n}\right\}$.

For this purpose we use the theorem on the extension of a function by continuity (see [1, Chapter I, §8.5]). Let $\tilde{w} \in \widetilde{D}_{K}^{H_{p} M_{p}}$ and let $\left\{w_{j}\right\} \subset \mathscr{D}_{K}^{*}$ be the sequence which converges to $\tilde{w}$ in $\widetilde{\mathscr{D}}_{K}^{H_{p} M_{p}}$. We shall prove that $F_{q}\left(w_{j}\right)$ converges in $(\mathscr{C B R})_{A}$ when $j \rightarrow \infty$, uniformly in $q \in \mathscr{N}_{0}^{n}$. To do this we shall show that $\left\{F_{q}\left(w_{j}\right), j \in \mathscr{N}\right\}$ is a Cauchy sequence in $(\mathscr{C} \mathscr{B})_{A}$, uniform in $q \in \mathscr{N}_{0}^{n}$.

Let $W$ be a neighbourhood of zero in $(\mathscr{C} \mathscr{B})_{A}$. Then it contains the ball $B(0, L) \subset(\mathscr{C} \mathscr{B})_{A}$ for some $L>0$. Consequently, $F_{q}\left(V_{\beta}\right) \subset W, q \in \mathscr{N}_{0}^{n}$, where $V_{\beta}$ is given by (3). Let $j_{0}$ be such that $w_{i}-w_{j} \in V_{\beta}, i, j \geq j_{0}$. Then

$$
F_{q}\left(w_{i}\right)-F_{q}\left(w_{j}\right)=F_{q}\left(w_{i}-w_{j}\right) \in W, \quad i, j \geq j_{0}, q \in \mathscr{N}_{0}^{n},
$$


and $F_{q}\left(w_{j}\right)$ converges to $h_{q}$ in $(\mathscr{C} \mathscr{B})_{A}$, when $j \rightarrow \infty$, uniformly in $q \in \mathscr{N}_{0}^{n}$. By the cited extension theorem it follows that $\bar{F}_{q}(\tilde{w})=h_{q} \in(\mathscr{C} \mathscr{B})_{A}, q \in \mathscr{N}_{0}^{n}$; every $\bar{F}_{q}, q \in \mathscr{N}_{0}^{n}$, is uniquely defined.

We shall prove that

$$
h_{q}=D^{q}(T \star \tilde{w}) / q ! L^{|q|}=\left(D^{q} T \star \tilde{w}\right) / q ! L^{|q|}, \quad q \in \mathscr{N}_{0}{ }^{n} .
$$

The sequence $\left\{w_{j}\right\}$ converges to $\tilde{w}$ in $\mathscr{E}_{B_{r}}^{\prime *}$, as well. Therefore $\left(T \star w_{j}\right)^{(q)}=$ $D^{q} T \star w_{j}$ converges to $D^{q} T \star \tilde{w}$ in $\mathscr{D}_{\Omega_{1}}^{\prime *}$, when $j \rightarrow \infty, q \in \mathscr{N}_{0}^{n}$ (see [6, Theorem 6.12]). Thus $\left(D^{q} T \star \tilde{w}\right) / q ! L^{|q|}$ must be $h_{q}, q \in \mathscr{N}_{0}^{n}$. Since the derivative is a continuous mapping of $\mathscr{D}_{\Omega_{1}}^{\prime *}$ into $\mathscr{D}_{\Omega_{1}}^{\prime *}$, it follows that $h_{q}=$ $D^{q}(T \star \tilde{w}) / q ! L^{|q|}$, as well. Consequently, $T * \tilde{w} \in \mathscr{A}\left(\Omega_{1}\right)$.

In the third step of the proof it remains only to use Theorem A. Let us remark that if $\varphi \in C^{\infty}$ has support in the compact set $Q \Subset K$ and $q_{H_{p} M_{p}}(\varphi)<\infty$, then $\varphi \in \widetilde{\mathscr{D}}_{K}^{H_{p} M_{p}}$. We also know that $\mathscr{A}(\omega) \subset \mathscr{E}^{*}(\omega)$ and that $P(D) \mathscr{E}^{*}(\omega) \subset \mathscr{E}^{*}(\omega)$ for any open set $\omega \subset \mathscr{R}^{n}$. According to the above remark we deduce from Theorem A that

$$
\frac{D^{q} T}{q ! L^{|q|}}=P(D) \frac{\left(\varphi \star D^{q} T\right)}{q ! L^{|q|}}+w \star D^{q} T \quad \text { on } \Omega, q \in \mathscr{N}_{0}^{n} .
$$

Therefore $T \in \mathscr{A}(\Omega)$.

\section{Applications of Theorem 1}

Direct consequences of Theorem 1 are:

1. Lemma 2.4 in [4] we know the analytic form of the operator $P(D)$ and of the functions $\varphi$ and $w$ given in Theorem A. With these $P(D), \varphi$ and $w$, Theorem 1 asserts that the equation $(P(D) X)(x)+(w \star f)(x)=f(x), x \in \Omega$, has a solution $X=(\varphi * f) \in \mathscr{A}(\Omega)$ for any $f \in \mathscr{A}(\Omega)$.

2. Denote by $\delta_{h}$ the distribution $\delta$ shifted in the point $h$. The function $H: h \rightarrow \delta_{h} \star T$ maps $\mathscr{R}^{n}$ into $\mathscr{D}^{\prime *}\left(\mathscr{R}^{n}\right)$ and has all derivatives. Theorem 1 asserts that $H$ is real analytic if and only if $T$ is defined by a real analytic function. The property that $H$ is real analytic means that the set $\left\{D^{q}\left(\delta_{h} \star T\right) / q ! L^{|q|}, q \in \mathscr{N}_{0}^{n}, h \in K\right\}$ is bounded in $D^{\prime *}\left(\mathscr{R}^{n}\right)$ for every compact set $K \subset \mathscr{R}^{n}$ and an $L>0$ which depends on $K$, namely that

$$
\sup _{h \in K, q \in \mathscr{N}_{0}^{n}}\left|D^{q}(T \star w)(h)\right| / q ! L^{|q|}<C \quad \text { for every } w \in \mathscr{D}^{*}\left(\mathscr{R}^{n}\right)
$$

where $C>0$ depends on $K$ and $w \in \mathscr{D}^{*}\left(\mathscr{R}^{n}\right)$.

Application of Theorem 1 to convolution equations. Let

$$
A \star T \equiv \sum_{k=1}^{m} A_{j, k} \star T_{k}, \quad j=1, \ldots, m,
$$

where $A=\left(A_{j, k}\right)$ is a given $(m \times m)$-matrix of elements belonging to $\mathscr{E}^{\prime *}\left(\mathscr{R}^{n}\right)$ and $T$ is an $m$-tuple $\left\{T_{1}, \ldots, T_{m}\right\}$ of unknown ultradistributions.

Theorem 2. Suppose that the system $T \star A=0$ has the following property: Any solution which belongs to $\left(\mathscr{E}^{*}\left(\Omega_{1}\right)\right)^{m}$ belongs to $\left(\mathscr{A}\left(\Omega_{1}\right)\right)^{m}$ as well. Then for 
every m-tuple $U$ of ultradistributions which is a solution of the system $T \star A=0$ there exists $r>0$ such that $U \in(\mathscr{A}(\Omega))^{m}, \Omega=\Omega_{1}-B_{r}$.

Proof. For the sake of simplicity we shall prove Theorem 2 only in case $m=1$. Suppose that $U \in \mathscr{D}^{\prime *}(\Omega)$ is a solution to equation $T \star A=0$. Then $U \star w \in$ $\mathscr{E}^{*}\left(\Omega_{1}\right)$ for every $w \in \mathscr{D}^{*}\left(\bar{B}_{r}\right)$ satisfies equation $T \star A=0$ as well, because of properties of the convolution. Since $U \star w \in \mathscr{E}^{*}\left(\Omega_{1}\right)$, by the supposition in Theorem $2, U \star w$ belongs to $\mathscr{A}\left(\Omega_{1}\right)$. Theorem 1 asserts that $U \in \mathscr{A}(\Omega)$. This is the end of the proof.

Application of Theorem 1 to Painlevês theorem. Let $V$ be an open set in $\mathscr{C}$ and $\Omega=V \cap \mathscr{R}$. Classical Painlevé's theorem asserts that a function $f$ holomorphic on $V \backslash \Omega$ and continuous on $V$ is holomorphic on the whole set $V$. It is well known that the continuity can be replaced by the existence and equality of the $\operatorname{limits} \lim _{y \rightarrow 0^{+}} f(x \pm i y)$ in $\mathscr{D}^{\prime}(\Omega)$. Theorem 1 admits to weaken this condition, supposing that the above limits exist in $\mathscr{D}^{\prime *}(\Omega)$.

Theorem 3. Let $f$ be a holomorphic function on $V \backslash \Omega$. If the limits $f(x \pm i 0)=$ $\lim _{y \rightarrow 0^{+}} f(x \pm i y)$ exist in $\mathscr{D}^{\prime *}(\Omega)$ and $f(x+i 0)=f(x-i 0)$, then $f$ is holomorphic on $V$.

Proof. The method of the proof is just the same as for distributions. First we have to apply classical Painlevé's theorem to the convolution $f \star w, w \in \mathscr{D}_{K}^{*}$, and then use Theorem 1.

\section{REFERENCES}

1. N. Bourbaki, Topologie général, Chapitres 1, 2, Hermann, Paris, 1951.

2. L. Hörmander, The analysis of linear partial differential operators. I, II, Springer-Verlag, Berlin, 1983.

3. A. Kaneko, Representation of hyperfunctions by measures and some of its applications, J. Fac. Sci. Univ. Tokyo Sect. IA Math. 19 (1992), 321-352.

4. H. Komatsu, Microlocal analysis in Gevrey class and in complex domains, Microlocal Analysis and Applications (L. Cattabriga and L. Rodino, eds.), Springer-Verlag, Berlin and Heidelberg, 1991.

5. __ Relative cohomology of sheaves of solutions of differential equations, Séminaire Lions (Schwartz, 1966), reprinted in Proceedings, pp. 190-259.

6. _ Ultradistributions I, J. Fac. Sci. Univ. Tokyo Sect. IA Math. 20 (1973), 23-105.

7. S. Pilipović, Characterisations of bounded sets in spaces of ultradistributions, Proc. Amer. Math. Soc. 120 (1994), 1191-1206.

8. L. Schwartz, Théorie des distributions. II, Hermann, Paris, 1951.

Institute of Mathematics, University of Novi Sad, 21000 Novi Sad, Yugoslavia 